\title{
Análise comparativa da qualidade de vida de adultos e idosos envolvidos com a prática regular de atividade física
}

\section{Comparative analysis of quality of life of adult and elderly involved in the practice of regular physical activity}

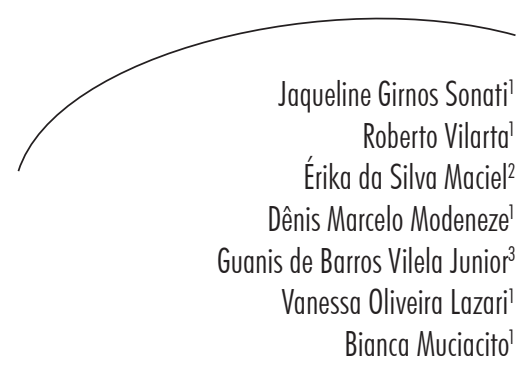

\section{Resumo}

Objetivo: Identificar diferenças na qualidade de vida de adultos e idosos, ambos praticantes de atividade física. Métodos: Foram estudados 117 sujeitos praticantes de atividade física, com idade entre 40 e 80 anos. Para a caracterização da amostra, foram analisadas as variáveis idade, sexo, renda familiar e escolaridade. Para a comparação entre os grupos de adultos e idosos, foram analisadas as variáveis peso, estatura, índice de massa corporal, domínios e facetas da qualidade de vida (Whoqol-Bref). O teste de Kolmogorov-Smirnov foi aplicado para análise da normalidade e o teste de MannWhitney, para a comparação de medidas contínuas ou ordenáveis entre dois grupos independentes; adotou-se valor de $\mathrm{p}<0,05$. Resultados: Foram encontradas diferenças significantes para os domínios psicológico, relações sociais e qualidade de vida geral, sendo que os idosos apresentaram os maiores valores médios. Conclusão: Idosos ativos, comparados aos adultos ativos, percebem melhor qualidade de vida nos aspectos da vida ativa, do desempenho das atividades da vida diária, da aceitação da aparência física, satisfação financeira e disponibilidade de tempo para as atividades de lazer.

\section{Abstract}

Objective: The study aimed to identify differences in the quality of life of elderly and adults engaged in physical activity. Methods: One hundred seventeen subjects engaged in physical activity, aged between 40 and 80 years, were investigated. To characterize the sample, the variables age, sex, income and educational level were analyzed. For comparison between groups of adults and the elderly were analyzed variables weight, height, body mass index, domains and facets of quality of life (Whoqol-Bref). The Kolmogorov-Smirnov test was applied to analyze normality, and the Mann-Whitney test compared continuous or sortable between two independent groups; the value adopted

Palavras-chave: Qualidade de Vida. Envelhecimento. Atividade Física. Idoso. Adulto.

Key words: Quality of Life. Aging. Physical Activity. Elderly. Adult.

\footnotetext{
Departamento de Atividade Física Adaptada, Faculdade de Educação Física. Universidade Estadual de Campinas. Campinas, SP, Brasil.

2 Departamento de Ciências e Tecnologia de Alimentos, Escola Superior de Agricultura "Luiz de Queiroz". Universidade de São Paulo. Piracicaba, SP, Brasil.

Programa de Pós-graduação em Educação Física. Universidade Metodista de Piracicaba. Piracicaba, SP, Brasil.

Correspondência / Correspondence

Jaqueline Girnos Sonati

E-mail: j.girnos@gmail.com
} 
was $\mathrm{p}<0.05$. Results: Significant differences were found for the psychological domain, social relationships domain and overall, and the elderly had the highest mean values. Conclusion: Active elderly, compared to active adults, have better perception of quality of life aspects from active life, the performance of activities of daily living, the acceptance of physical appearance, financial satisfaction and time availability for leisure activities.

\section{INTRODUÇÃO}

A prática da atividade física tem sido orientada para o aprimoramento de funções fisiológicas dos sistemas cardiovascular e musculoesquelético, ${ }^{1,2}$ bem como para incentivar maior participação social, em especial para grupos de idosos em atividades de lazer e nas agremiações conhecidas como as universidades da terceira idade ou nos grupos de adultos envolvidos com atividade esportivas e culturais. ${ }^{3,4}$

Ampla é a gama de estudos desenvolvidos para obter maior compreensão do impacto de agravos e doenças sobre a qualidade de vida; no entanto, poucos deles têm associado ações específicas dirigidas à promoção da saúde com os hábitos da vida moderna e a qualidade de vida.

A adoção de hábitos de vida saudável, como a prática de atividade física, promove a saúde ao longo da vida, influenciando o envelhecimento de maneira positiva ${ }^{5-8}$ e a melhor percepção da qualidade de vida que contempla aspectos multifatoriais das facetas físicas, psicológicas, sociais e ambientais.?

Estudos mostram que o idoso com baixo nível de atividade pode se tornar frágil e dependente e que a atividade física sistematizada colabora para sua independência e melhora sua percepção de qualidade de vida. ${ }^{10-12}$

Para o adulto, a prática da atividade física está associada com a percepção positiva da qualidade de vida, principalmente nos aspectos do domínio físico. Esse domínio se relaciona com a capacidade para o trabalho, energia para as atividades do dia a dia e locomoção. ${ }^{13}$ Assim, a percepção da qualidade de vida pode ser considerada relevante e amplo indicador relacionável com domínios que compõem o conceito de saúde, inclusive subsidiando estudos voltados para a definição de estratégias e ações de promoção da saúde em intervenções populacionais específicas. ${ }^{14}$

Apesar da complexidade inerente ao termo "qualidade de vida", que inclui aspectos culturais, valores, objetivos, expectativas e preocupações individualizadas, esse conceito pode ter valor pragmático dentro de contextos específicos em estudos populacionais, ou mesmo nas abordagens pontuais da pesquisa clínica ou aquelas dirigidas ao conhecimento das diferenças comportamentais entre grupos etários. ${ }^{15}$

Estudos demonstram que os adultos relacionam melhor qualidade de vida em função da possibilidade de desenvolver atividades laborais, gerar renda e manter o equilíbrio das finanças que permitam atender às suas expectativas pessoais e familiares. ${ }^{16,17}$ Os idosos, no entanto, relatam melhor qualidade de vida vinculada às relações sociais cultivadas nas reuniões com amigos ou família, pois esse contato aumenta suas percepções de pertencimento, visibilidade social, atividade produtiva e valorização pessoal, por se sentirem necessários na comunidade. ${ }^{3,18}$

O objetivo deste estudo, portanto, foi identificar as diferenças entre as percepções na qualidade de vida de adultos e idosos, ambos praticantes de atividade física.

\section{MÉTODOS}

\section{Sujeitos}

O estudo teve características do tipo transversal, de amostra não probabilística e de conveniência, com projeto aprovado pelo Comitê de Ética em Pesquisa da Universidade Estadual 
de Campinas (UNICAMP), sob nos 622/2008, $789 / 2008$ e 1039/2009. O período do estudo se deu entre os anos de 2009 e 2010.

Foram avaliados 179 indivíduos adultos e idosos praticantes de atividade física, dos quais 117 atenderam aos critérios de inclusão: idade de 40 anos ou mais; realizar, no mínimo, 30 minutos de atividade física sistematizada pelo menos cinco vezes na semana; preencher corretamente os questionários e assinar $\mathrm{O}$ Termo de Consentimento Livre e Esclarecido. Os sujeitos adultos frequentavam as aulas de hidroginástica na Faculdade de Educação Física da Universidade Estadual de Campinas (UNICAMP) ou praticavam bike indoor em duas academias da mesma cidade. Já os idosos frequentavam as aulas de ginástica e caminhadas na Universidade Aberta à Terceira Idade (UnATI) na Escola Superior de Agricultura "Luiz de Queiroz" da Universidade de São Paulo (USP), na cidade de Piracicaba.

A amostra foi classificada em duas faixas etárias: a primeira incluiu indivíduos entre 40 e 59 anos $(n=56)$ e a segunda com 60 anos ou mais $(\mathrm{n}=61)$. Todos foram orientados quanto ao preenchimento dos questionários, sendo os mesmos autorrespondidos. Para caracterização socioeconômica da amostra, foi utilizado um questionário com as variáveis "idade", "sexo", "renda familiar" e "escolaridade" e para as características antropométricas e de composição corporal as medidas de peso corporal (PC), estatura e índice de massa corporal (IMC).

\section{Qualidade de vida}

Para a avaliação da qualidade de vida, foi utilizado o instrumento World Health Organization Quality of Life Assessment (WhoqolBref), desenvolvido pela Organização Mundial da Saúde (OMS) e validado para a população brasileira. ${ }^{19}$ Esse instrumento é composto de 26 questões, sendo duas questões sobre a qualidade de vida geral; sete questões que compõem o domínio físico; seis, o domínio psicológico; três, o domínio relações sociais; e oito, o domínio meio ambiente. Foram analisados os domínios e consideradas as questões de maneira independente. Considerou-se a variabilidade do escore de 4 a 20 para os domínios e de 1 a 5 para as facetas. Valores entre 15 e 20 (domínios) e entre 4 e 5 (facetas) são considerados percepções melhores de qualidade de vida.

\section{Medidas antropométricas}

O peso corporal (PC) foi medido em quilogramas $(\mathrm{kg})$, sendo utilizada para isso balança digital da marca Healthmeter com capacidade de até $150 \mathrm{Kg}$; a estatura foi medida em centímetros (cm), com auxílio do estadiômetro de parede da marca Sanny; e foi calculado o índice de massa corporal (IMC) para verificação do estado nutricional. Os critérios para a classificação do estado nutricional são os propostos pela OMS. ${ }^{20}$

\section{Análise estatística}

Os dados foram analisados pelo programa estatístico SPSS 10.01, sendo efetuada análise descritiva para dados absolutos e valores relativos, média, desvio-padrão, mediana e variância. $\mathrm{O}$ teste de Kolmogorov-Smirnov foi aplicado para análise da normalidade dos dados, onde as variáveis foram consideradas não paramétricas $(\mathrm{p} \leq 0,05)$. Assim, foi utilizado o teste de MannWhitney para a comparação de medidas contínuas ou ordenáveis entre os dois grupos independentes, adotando-se valor de $\mathrm{p} \leq 0,05$.

\section{RESULTADOS}

O grupo de adultos foi predominantemente composto de mulheres (88\%) e apresentou médias de 47,43 $( \pm 4,87)$ anos de idade, PC de $72,86( \pm 14,16) \mathrm{kg}$, estatura de 163,67 $( \pm 9,63)$ $\mathrm{cm}$ e IMC médio de 27,27 $( \pm 5,37)$, indicando prevalência de sobrepeso nos adultos. $\mathrm{O}$ grupo de idosos também teve predominância do gênero feminino $(86 \%)$ e médias de $68,08( \pm 4,73)$ anos 
de idade, 69,42 ( $\pm 11,89) \mathrm{kg}$ de PC, 158,86 $\mathrm{cm}$ de estatura e IMC de 27,46 ( $\pm 4,20)$, caracterizando também sobrepeso. Houve diferença significante entre os grupos quanto às variáveis "idade" e "estatura" (tabela 1).

O estudo das características socioeconômicas dos grupos de adultos e idosos mostrou que $57,14 \%$ dos adultos e 78,68\% dos idosos eram naturais do interior do Estado de São Paulo;
$69,64 \%$ dos adultos e $75,4 \%$ dos idosos relataram estar casados; $82,14 \%$ dos adultos e 9,83\% dos idosos atuavam no mercado de trabalho; $98 \%$ dos adultos e 74\% dos idosos tinham mais de 11 anos de estudos; e 62,5\% dos adultos e $31,14 \%$ dos idosos referiram receber mais que oito salários mínimos por mês. O percentual de resposta dos idosos referente à renda foi baixo, sendo calculado somente em cima daqueles que responderam.

Tabela 1. Análise descritiva das variáveis antropométricas de adultos $(\mathrm{n}=56)$ e idosos ativos $(\mathrm{n}=61)$. Campinas-SP, 2009-2010.

\begin{tabular}{lccccc}
\hline \multicolumn{1}{c}{ Variáveis } & \multicolumn{2}{c}{ Adultos } & \multicolumn{2}{c}{ Idosos } & p valor \\
& média $\pm \mathrm{dp}$ & mediana & média $\pm \mathrm{dp}$ & mediana & \\
\hline Idade (anos) & $47,43 \pm 4,87$ & 46,00 & $68,08 \pm 4,73$ & 68,00 & $<0,0001^{*}$ \\
Peso corporal (kg) & $72,86 \pm 14,16$ & 67,70 & $69,42 \pm 1,89$ & 69,40 & 0,2747 \\
Estatura (m) & $163,67 \pm 9,63$ & 163,00 & $158,86 \pm 7,04$ & 158,75 & $0,0029 *$ \\
IMC $\left(\mathrm{kg} / \mathrm{m}^{2}\right)$ & $27,27 \pm 5,37$ & 25,94 & $27,46 \pm 4,20$ & 27,17 & 0,3861 \\
\hline
\end{tabular}

*Diferença significante teste Mann-Whitney; IMC= índice de massa corporal.

A análise das questões que compõem a qualidade de vida, como mostra a tabela 2, demonstrou diferença significante entre os grupos nas questões 1 (Como você avaliaria sua qualidade de vida?), 4 (O quanto você precisa de algum tratamento médico para levar sua vida diária?), 11 (Você é capaz de aceitar sua aparência física?), 12 (Você tem dinheiro suficiente para satisfazer suas necessidades?), 14 (Em que medida você tem oportunidades de atividade de lazer?), 17 (Quão satisfeito[a] você está com sua capacidade de desempenhar as atividades do seu dia a dia?), 19 (Quão satisfeito[a] você está consigo mesmo?), 20 (Quão satisfeito[a] você está com suas relações pessoais [amigos, parentes, conhecidos, colegas]?) e 21 (Quão satisfeito[a] você está com sua vida sexual?). O grupo de idosos apresentou as maiores médias, com exceção somente da questão 4, que apresentou maior média para o grupo de adultos. 
Tabela 2. Diferenças encontradas entre as facetas da qualidade de vida de adultos $(n=56)$ e idosos ativos (n=61). Campinas-SP, 2009-2010.

\begin{tabular}{|c|c|c|c|c|c|}
\hline Domínios & Q & Grupos & Média $\pm \mathrm{dp}$ & $\mathrm{p}$ valor & Mediana \\
\hline \multirow[t]{4}{*}{ Físico } & 4 & Adultos & $4,10 \pm 0,98$ & $0,0101^{*}$ & 4,00 \\
\hline & & Idosos & $3,59 \pm 1,08$ & & 4,00 \\
\hline & 17 & Adultos & $3,71 \pm 0,96$ & $0,0232^{*}$ & 4,00 \\
\hline & & Idosos & $4,13 \pm 0,71$ & & 4,00 \\
\hline \multirow[t]{2}{*}{ Psicológico } & 11 & Adultos & $3,53 \pm 1,00$ & $0,0039 *$ & 3,00 \\
\hline & & Idosos & $4,06 \pm 0,70$ & & 4,00 \\
\hline \multirow[t]{2}{*}{ Psicológico } & 19 & Adultos & $3,53 \pm 1,06$ & $0,0016^{*}$ & 4,00 \\
\hline & & Idosos & $4,14 \pm 0,74$ & & 4,00 \\
\hline \multirow[t]{2}{*}{ Relações sociais } & 20 & Adultos & $3,80 \pm 1,01$ & $0,0249 *$ & 4,00 \\
\hline & & Idosos & $4,26 \pm 0,60$ & & 4,00 \\
\hline \multirow[t]{2}{*}{ Relações sociais } & 21 & Adultos & $3,10 \pm 1,23$ & $0,0447 *$ & 3,00 \\
\hline & & Idosos & $3,59 \pm 0,86$ & & 4,00 \\
\hline \multirow[t]{2}{*}{ Meio ambiente } & 12 & Adultos & $3,07 \pm 0,96$ & $0,0008^{*}$ & 3,00 \\
\hline & & Idosos & $3,70 \pm 0,91$ & & 4,00 \\
\hline \multirow[t]{2}{*}{ Meio ambiente } & 14 & Adultos & $3,16 \pm 1,07$ & $0,0343^{*}$ & 3,00 \\
\hline & & Idosos & $3,60 \pm 0,88$ & & 4,00 \\
\hline \multirow[t]{2}{*}{ Geral } & 1 & Adultos & $3,75 \pm 0,83$ & $0,0307 *$ & 4,00 \\
\hline & & Idosos & $4,11 \pm 0,55$ & & 4,00 \\
\hline
\end{tabular}

*Diferença significante teste Mann-Whitney; Q= número da questão. 
A análise das médias dos domínios que compõem a qualidade de vida mostrou os maiores valores para o grupo com idade acima de 60 anos e com diferença significante para o domínio psicológico e relações sociais (tabela 3).

Tabela 3. Comparação entre os domínios da qualidade de vida de adultos ( $\mathrm{n}=56)$ e idosos ativos $(\mathrm{n}=61)$. Campinas-SP, 2009-2010.

\begin{tabular}{lccccc}
\hline \multicolumn{1}{c}{ Domínios } & $\begin{array}{c}\text { Adultos } \\
\text { média } \pm \mathrm{dp}\end{array}$ & Mediana & $\begin{array}{c}\text { Idosos } \\
\text { média } \pm \mathrm{dp}\end{array}$ & Mediana & p valor \\
\hline Físico & $15,49 \pm 2,79$ & 15,71 & $16 \pm 2,21$ & 16,00 & 0,394 \\
Psicológico & $14,65 \pm 2,63$ & 15,33 & $15,86 \pm 1,76$ & 16,00 & $0,011^{*}$ \\
Relações sociais & $14,24 \pm 3,34$ & 14,67 & $15,95 \pm 2,22$ & 16,00 & $0,005^{*}$ \\
Meio ambiente & $14,65 \pm 2,50$ & 15,00 & $15,59 \pm 2,34$ & 15,50 & 0,086 \\
Geral & $14,59 \pm 3,35$ & 16,00 & $16,00 \pm 2,23$ & 16,00 & $0,030^{*}$ \\
\hline
\end{tabular}

*Diferença significante pelo teste Mann-Whitney.

\section{DISCUSSÃO}

A falta de habilidades para a vida diária, a falta de energia e a presença de dor são aspectos do domínio físico que parecem ser importantes para a qualidade de vida. ${ }^{21}$ Esses aspectos influenciam a percepção da qualidade de vida durante o processo de envelhecimento, pois ocorrem mudanças corporais importantes, dentre as quais a perda da massa livre de gordura e o aumento da gordura corporal com distribuição central. ${ }^{22-25}$ Sabe-se que a prática de exercícios físicos contribui para a melhora desses fatores, refletindo positivamente na qualidade de vida. ${ }^{4,14,26-28}$ Essas observações são também sustentadas pela OMS, ao considerar diretrizes para o estabelecimento de políticas para o envelhecimento ativo. ${ }^{29}$

O estudo mostrou que mesmo entre grupos que fazem exercício físico há diferença significante na qualidade de vida geral, quando se comparam adultos e idosos: indivíduos acima de 60 anos mostram os maiores escores, indicando que a qualidade de vida de indivíduos ativos pode estar relacionada com a faixa etária. De acordo com Peel et al., ${ }^{30}$ os determinantes de um envelhecimento saudável são a prática regular da atividade física, IMC dentro da normalidade e estilo de vida saudável, aspectos presentes na vida dos idosos pesquisados que frequentam a Universidade Aberta da Terceira Idade (UTA). Segundo Alexandre et al., ${ }^{27}$ idosos que frequentam a UTA fazem atividade física, mantêm relacionamento social e melhoram sua intelectualidade.

A análise comparativa do domínio físico não apresentou diferença significante entre os dois grupos. Já a análise por facetas desse domínio mostrou a importância de auxílio médico para manter as atividades diárias no grupo de idosos, condição esperada no processo 
de envelhecimento quando as comorbidades aparecem de maneira mais acentuada. Vale lembrar que a saúde pode ser um bom indicador de qualidade de vida, mas é insuficiente para atestar o sucesso do envelhecimento. ${ }^{31}$ Dentro do domínio físico, a faceta sobre a satisfação em desempenhar as atividades da vida diária também apresentou diferença significante entre os grupos, sendo o maior escore para os idosos. Esse aspecto também foi observado por Robinson, ${ }^{32}$ mostrando que independência, liberdade e habilidade para se mover são aspectos importantes para o trabalho e a inserção do idoso na comunidade. Já para o adulto que ainda está no mercado de trabalho, esse aspecto talvez seja influenciado pela expectativa gerada sobre a habilidade de executar ou não as tarefas laborais que lhe são determinadas.

O domínio psicológico apresentou diferença significante entre os dois grupos, mais evidentes nas questões da aceitação da aparência física e da satisfação com si mesmo, em que adultos têm menores escores. Matsuo ${ }^{33}$ verificou em seu estudo que as idosas ativas percebiam a imagem corporal desejada mais próxima da imagem corporal real, quando comparadas com idosas não ativas, mostrando maior aceitação de sua aparência física. Para o adulto, as questões sobre satisfação com o corpo remetem a cobranças sociais que exigem perfis antropométricos cada vez mais magros, ${ }^{34,35}$ e podem ser relacionadas com a aceitação social e o sucesso profissional, gerando constante insatisfação com a aparência física.

Quanto ao domínio das relações sociais, observou-se diferença significante na comparação entre os grupos, sendo as relações pessoais e a satisfação com a vida sexual as mais valorizadas pelos idosos. Segundo Moraes \& Souza, ${ }^{16}$ as relações pessoais são fatores que se associam positivamente ao sucesso do envelhecimento, juntamente com a independência para realização das tarefas diárias e a autonomia. Essa situação, encontrada no presente estudo, leva a pensar na atividade física como instrumento social ${ }^{36}$ para a terceira idade. A vida sexual mostrou ser importante para os dois grupos, com escores médios maiores que os observados em outros trabalhos. ${ }^{21,37}$

O domínio referente ao meio ambiente, apesar de não apresentar diferença significante, mostrou que os adultos referem que as questões financeiras ainda estão ligadas à formação familiar e ao desejo da conquista de bens materiais - ou seja, os adultos ainda buscam a estabilidade financeira, condição relacionada com a falta de tempo para atividades de lazer e insatisfação financeira, ao contrário dos idosos, que possuem condição inversa. Esses achados podem ser sustentados por estudos que relacionaram a alta escolaridade e o bom nível socioeconômico a maiores escores para o domínio meio ambiente. ${ }^{14,27}$

É importante salientar que, neste estudo, decidiu-se optar por um instrumento único para a avaliação da percepção da qualidade de vida dos grupos, padronizando o método, já que o questionário Whoqol-Bref demostrou consistência interna, validade concorrente, validade discriminante e validade de critério satisfatório em amostras com idosos brasileiros. ${ }^{38}$

\section{CONCLUSÃO}

Oestudo mostrou que o grupo de idosos referiu melhor percepção de qualidade de vida associada aos aspectos da vida ativa, desempenho das atividades da vida diária, aceitação da aparência física, satisfação financeira e disponibilidade de tempo para as atividades de lazer mostrando que, para esse grupo, o processo de envelhecimento não afetou de maneira negativa a percepção da qualidade de vida.

Já para o grupo de adultos, notou-se que fatores importantes como a atividade laboral e a família podem estar influenciando a percepção da qualidade de vida desses indivíduos, sugerindo a necessidade de um estudo de caráter longitudinal para o acompanhamento das mudanças ao longo dos anos e também para a identificação das variáveis de confundimento. 


\section{REFERÊNCIAS}

1. Häkkinen A, Rinne M, Vasankari T, Santtila M, Häkkinen K, Kyröläinen H. Association of physical fitness with health-related quality of life in Finnish young men. Health Qual Life Outocomes 2010;8:1-8.

2. Sloan RA, Sawada SS, Martin CK, Church T, Blair $\mathrm{SN}$. Associations between cardiorespiratory fitness and health-related quality of life. Health Qual Life Outcomes 2009;7:1-5. DOI: 10.1186/1477-7525-8-15.

3. Sonati J, Modeneze DM, Vilarta R, Maciel ES, Boccaletto EMA, Da Silva CC. Body composition and quality of life (QoL) of the elderly offered by the "University Third Age" (UTA) in Brazil. Arch Gerontol Geriatr 2011;52(1):e31-35.

4. Silva RS, Da Silva I, Da Silva RA, Souza L, Tomasi E. Physical activity and quality of life. Ciênc Saúde Coletiva 2010;15(1):115-20.

5. MacAuley E, Bane SM, Rudolph DL, Lox CL Physique anxiety and exercise in middle-aged adults. J Gerontol Ser B, Psychol Sci Soc Sci 1995;50(5):229-35.

6. Rejeski WJ, Mihalko SL. Physical activity and quality of life in older adults. J Gerontol Ser A, Biol Sci Med Sci 2001;56(2 Suppl ):23-35.

7. Lavie CJ, Milani RV. Disparate effects of improving aerobic exercise capacity and quality of life after cardiac rehabilitation in young and elderly coronary patients. J Cardiopulm Rehabil 2000;20(4):235-40.

8. Ai S, Koichiro O, Yoshio N, Isao M. Recommended level of physical activity and health-related quality of life among japanese adults. Health Qual Life Outcomes 2007;5:1-8.

9. WHOQOL Group. The World Health Organization Quality of Life assessment (WHOQOL): position paper from the World Health Organization. Soc Sci Med 1995;41(10):1403-09.

10. Grimby G. Muscle performance and structure in the elderly as studied cross-sectionally and longitudinally. J Gerontol Ser A, Biol Sci Med Sci 1995;50( ${ }^{\circ}$ Spec):17-22.

11. Cress ME, Buchner DM, Prohaska T, Rimmer J, Brown M, Macera C, et al. Physical activity programs and behavior counseling in older adult populations. Med Sci Sports Exerc 2004;36(11):1997-2003.

12. Pedrinelli A, Garcez-Leme LE, Nobre RSA. O efeito da atividade física no aparelho locomotor do idoso. Rev Bras Ortop 2009;44(2):96-101.

13. Maciel ES, Vilarta R, Modeneze DM, Sonati JG, Vasconcelos JS, Vilela GB Junio, et al. The relationship between physical aspects of quality of life and extreme levels of regular physical activity in adults. Cad Saúde Pública 2013;29(11):2251-60.
14. Paskulin L, Vianna L, Molzahn AE. Factors associated with quality of life of Brazilian older adults. Int Nurs Rev 2009;56(1):109-15.

15. Camfield L, Skevington S. On subjective well-being and quality of life. J Health Psychol 2008;13(6):764-75. DOI: $10.1177 / 1359105308093860$

16. De Moraes JFD, Souza VBA. Factors associated with the successful aging of the socially-active elderly in the metropolitan region of Porto Alegre. Rev Bras Psiquiatr 2005;27(4):302-08.

17. Vecchia RD, Ruiz T, Bocchi SCM, Corrente JE. Qualidade de vida na terceira idade: um conceito subjetivo. Rev Bras Epidemiol 2005;8(3):246-52.

18. Wang H, Karp A, Winblad B, Fratiglioni L. Latelife engagement in social and leisure activities is associated with a decreased risk of dementia: a longitudinal study from the Kungsholmen Project. Am J Epidemiol 2002;155(12):1081-87.

19. Fleck MPA, Louzada S, Xavier M, Chachamovich E, Vieira G, Santos L, et al. Application of the Portuguese version of the abbreviated instrument of quality life Whoqol-Bref. J Public Health 2000;34(2):178-83.

20. WHO;FAO Expert Consultation. Diet, Nutrition and the prevention of chronic diseases. Geneve: WHO; 2003. (Technical Report Series, n. 916).

21. Molzahn A, Skevington SM, Kalfoss M, Makaroff KS. The importance of facets of quality of life to older adults: an international investigation. Quality Life Res 2010;19:293-98. DOI: 10.1007/s11136-009-9579-7.

22. Fiatarone MA, Marks EC, Ryan ND, Meredith CN, Lipsitz LA, Evans WJ. High-intensity strength training in nonagenarians: effects on skeletal muscle. J Am Med Assoc 1980;263(22);3029-34.

23. Visser M, Langlois J, Guralnik JM, Cauley JA, Kronmal RA, Robbins, et al. High body fatness, but not low fat-free mass, predicts disability in older men and women: the Cardiovascular Health Study. Am J Clin Nutr 1998;68(3):584-90.

24. Matsudo SM, Matsudo VKR, Barros Neto TL. The impact of aging on anthropometric, neuromotor, and metabolic variables of physical fitness. Rev Bras Ciênc Mov 2000;8(4):21-32.

25. Fantin F, Di Francesco V, Fontana G, Zivelonghi A, L Bissoli, Zoico E, et al Longitudinal body composition changes in old men and women: interrelationships with worsening disability. J Gerontol Ser A, Biol Sci Med Sci 2007;62(12):1375-81.

26. Sonati JG, Modeneze DM, Vilarta R, Maciel ES Boccaletto EM. Body weight as an indicator of fat-free mass in active elderly women. Maturitas 2011;68(4):378-81. 
27. Alexandre TS, Cordeiro RC, Ramos LR. Factors associated to quality of life in active elderly. Rev Saúde Pública 2009;43(4):613-21.

28. Riesco E, Tessier S, Pérusse F, Turgeon S, Tremblay A, Weisnage J, et al. Impact of walking on eating behaviors and quality of life of premenopausal and early postmenopausal obese women. Menopause 2010;17(3):529-38.

29. World Health Organization. Active ageing: A policy framework [Internet]. Geneva: WHO; 2002 [Acesso em 2012 dez 12]. Disponível em: Http://whqlibdoc. who.int/hq/2002/who_nmh_nph_02.8.pdf

30. Peel NM, McClure RJ, Bartlett HP. Behavioral determinants of healthy aging. Am J Prev Med 2005;28(3):298-304. DOI 10.1016/j.amepre.2004.

31. Xavier FMF, Ferraz MPT, Marc N, Escosteguy NU, Moriguchi EH. Elderly people's definition of quality of life. Rev Bras Psiquiatr 2003;25(1);31-9.

32. Robinson J. Older adults and sexuality: The relationship to quality of life. [Masters Thesis]. British Columbia: University of Victoria; 2004.
33. Matsuo RF, Velardi M, Brandão MRF, Miranda MLJ. Imagem corporal de idosas e atividade física. Rev Mackenzie Educ Fís Esporte 2007;6(1):37-43.

34. Instituto de Nutrição Annes Dias. Obesidade e Desnutrição: projeto com gosto de saúde [Internet]. Rio de Janeiro: INAD; 2004 [Acesso em 10 mar 2010]. Disponível em: http: \|www.saude.rio.rj.gov.br

35. Schwartz MB, Brownell KD. Obesity and body image. Body Image 2004; 1(1):43-56.

36. Laberg S. Toward an integration of gender into Bourdieu's concept of cultural capital. Sociol Sport J 1995;12(2);132-46.

37. Skevington SM. Qualities of life, educational level and human development: an international investigation of health. Soc Psychiatr Psychiatr Epidemiol 2009;45(10);999-1009. DOI: 10.1007/s00127-009-0138-x.

38. Chachamovich E. Qualidade de vida em idosos desenvolvimento e aplicação do módulo WHOQOLOLD e teste do desempenho do instrumento Whoqol-Bref em uma população idosa brasileira [dissertação]. Porto Alegre: Universidade Federal do Rio Grande do Sul, Faculdade de Medicina; 2005. 